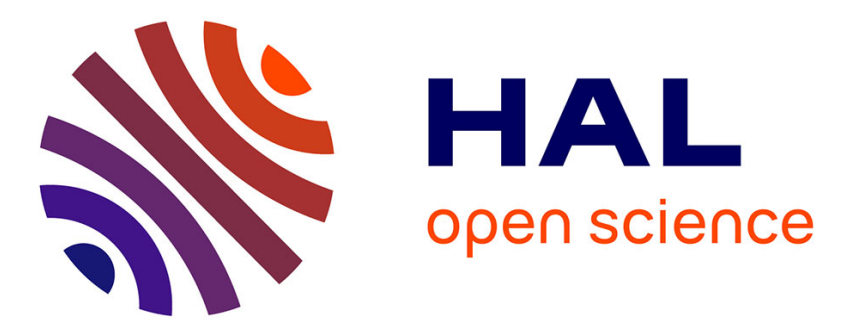

\title{
The limit case response of the archetypal oscillator for smooth and discontinuous dynamics
}

Qingjie Cao, Marian Wiercigroch, Ekaterina E Pavlovskaia, Celso Grebogi, J

Michael T Thompson

\section{- To cite this version:}

Qingjie Cao, Marian Wiercigroch, Ekaterina E Pavlovskaia, Celso Grebogi, J Michael T Thompson. The limit case response of the archetypal oscillator for smooth and discontinuous dynamics. International Journal of Non-Linear Mechanics, 2008, 43 (6), pp.462. 10.1016/j.ijnonlinmec.2008.01.003 . hal-00501777

\section{HAL Id: hal-00501777 \\ https://hal.science/hal-00501777}

Submitted on 12 Jul 2010

HAL is a multi-disciplinary open access archive for the deposit and dissemination of scientific research documents, whether they are published or not. The documents may come from teaching and research institutions in France or abroad, or from public or private research centers.
L'archive ouverte pluridisciplinaire HAL, est destinée au dépôt et à la diffusion de documents scientifiques de niveau recherche, publiés ou non, émanant des établissements d'enseignement et de recherche français ou étrangers, des laboratoires publics ou privés. 


\section{Author's Accepted Manuscript}

INTERNATIONAL JOURNAL OF

NON-LINEAR

MECHANICS

The limit case response of the archetypal oscillator for smooth and discontinuous dynamics

Qingjie Cao, Marian Wiercigroch, Ekaterina E Pavlovskaia, Celso Grebogi, J Michael T Thompson

PII: $\quad$ S0020-7462(08)00017-6

DOI: doi:10.1016/j.ijnonlinmec.2008.01.003

Reference: NLM 1443

To appear in: International Journal of NonLinear Mechanics

Received date: 2 November 2007

Revised date: 11 January 2008

Accepted date: 11 January 2008

Cite this article as: Qingjie Cao, Marian Wiercigroch, Ekaterina E Pavlovskaia, Celso Grebogi and J Michael T Thompson, The limit case response of the archetypal oscillator for smooth and discontinuous dynamics, International Journal of Non-Linear Mechanics (2008), doi:10.1016/j.ijnonlinmec.2008.01.003

This is a PDF file of an unedited manuscript that has been accepted for publication. As a service to our customers we are providing this early version of the manuscript. The manuscript will undergo copyediting, typesetting, and review of the resulting galley proof before it is published in its final citable form. Please note that during the production process errors may be discovered which could affect the content, and all legal disclaimers that apply to the journal pertain. 


\title{
The limit case response of the archetypal oscillator for smooth and discontinuous dynamics
}

\author{
Qingjie $\mathrm{Cao}^{a}$, Marian Wiercigroch $^{a}$, Ekaterina E Pavlovskaia ${ }^{a *}$, Celso Grebogi $^{a}$ and \\ J Michael T Thompson ${ }^{a, b}$ \\ ${ }^{a}$ Centre for Applied Dynamics Research, \\ King's College, Aberdeen AB24 3UE, Scotland, UK \\ ${ }^{b}$ Department of Applied Mathematics and Theoretical Physics, University of \\ Cambridge, Cambridge, CB3 0WA, UK
}

January 11, 2008

\begin{abstract}
In this paper, the limit case of the SD (smooth and discontinuous) oscillator is studied. This system exhibits standard dynamics governed by the hyperbolic structure associated with the stationary state of the double-well. The substantial deviation from the standard dynamics is the non-smoothness of the velocity in crossing from one well to another, caused by the loss of local hyperbolicity due to the discontinuity. Without dissipation, the KAM structure on the Poincare section is constructed with generic KAM curves and a series of fixed points associated with surrounded islands of quasi-periodic orbits and the chaotic connection orbits. It is found that, for a fixed set of parameters, a special chaotic orbit exits there which fills a finite region and connects a series of islands dominated by different chains of fixed points. As one adds weak dissipation, the periodic solutions in this finite region remain unchanged while the quasi-periodic solutions (isolated islands) are converted to the corresponding periodic solutions. The relevant dynamics for the system with weak dissipation under external excitation is shown having period doubling bifurcation leading to chaos, and multi-stable solutions.
\end{abstract}

keywords: SD oscillator, discontinuity, KAM structure, chaotic sea

\section{Introduction}

Discontinuous dynamical systems have attracted a lot of interest from the nonlinear dynamics community both in terms of theory, see for example [1, 2], and its

\footnotetext{
*Corresponding author's e-mail: e.pavlovskaia@abdn.ac.uk
} 
application $[3,4,5]$. Various types of discontinuities reported and studied in the literature are: Filippov-type discontinuity, the discontinuous right hand side [6], typical of dry friction dampers $[7,8]$, phase state discontinuity, such as impacting systems $[9,10]$, and also the system with non-smooth Jacobian matrix, such as the piecewise linearity[11, 12].

Discontinuous dynamical systems are of wide applicability both in engineering and mathematics, and a significant amount of work has been done to analyse the behaviour of these systems. Di Bernardo and co-workers $[13,14,15]$ studied the bifurcations for the sliding system with a border collision bifurcation. Banerjee et al. $[16,17]$ investigated bifurcations of the discontinuous maps for a power circuit system with discontinuous switches. Whiston [18] examined the singularities of a vibro-impact system. These studies range from theoretical formulation, methodology to the applications in engineering.

The motivation of this paper is to study the limit discontinuous case of the smooth and discontinuous (SD) oscillator introduced in [19] and modelled as a piecewise linear system in [20]. The SD oscillator is derived from an arch model which was first proposed by Thompson and Hunt, 1973 in [21], where stability of the arch was investigated by the energy method. This SD oscillator shown in Fig. 1a is comprised of a mass moving vertically and linked by a pair of inclined springs to rigid supports. The springs are assumed to be capable of resisting both tension and compression. This model is widely used in engineering, see [22, 23]. In [20], we introduced a trilinear model to investigate the SD oscillator analytically. The current study is focussed on the nonlinear behaviour of the system in the limiting discontinuous case, where the influence of weak dissipation and external excitation on the system dynamics is investigated.

This paper is organised as follows. In Section 2, the static response analysis is given for the archetypal oscillator representing the arch model showing the physical characteristics. In Section 3, conceptual response analysis and the physical model are established and the equations of motion are derived and formulated for the limit case of the archetypal SD oscillator. In Section 4, standard dynamics of the equilibrium state hyperbolicity of the double-well and the loss of local hyperbolicity at the saddle-like equilibrium for the unperturbed system is presented. In Section 5, numerical results for the system with weak dissipation and external excitation are presented showing relevant dynamics, co-existence of attractors, period doubling bifurcation leading to chaos and various harmonic responses.

\section{Static Response of the SD oscillator}

In this paper we consider the limit case for the smooth and discontinuous oscillator introduced in $[19,20]$. The arch model presented there has some physical characteristics, but the assumed response of the idealised springs is far from physical, especially in the limit case which is examined here. So it is better to think of it as a conceptual model. In spite of that, the results obtained by the dynam- 

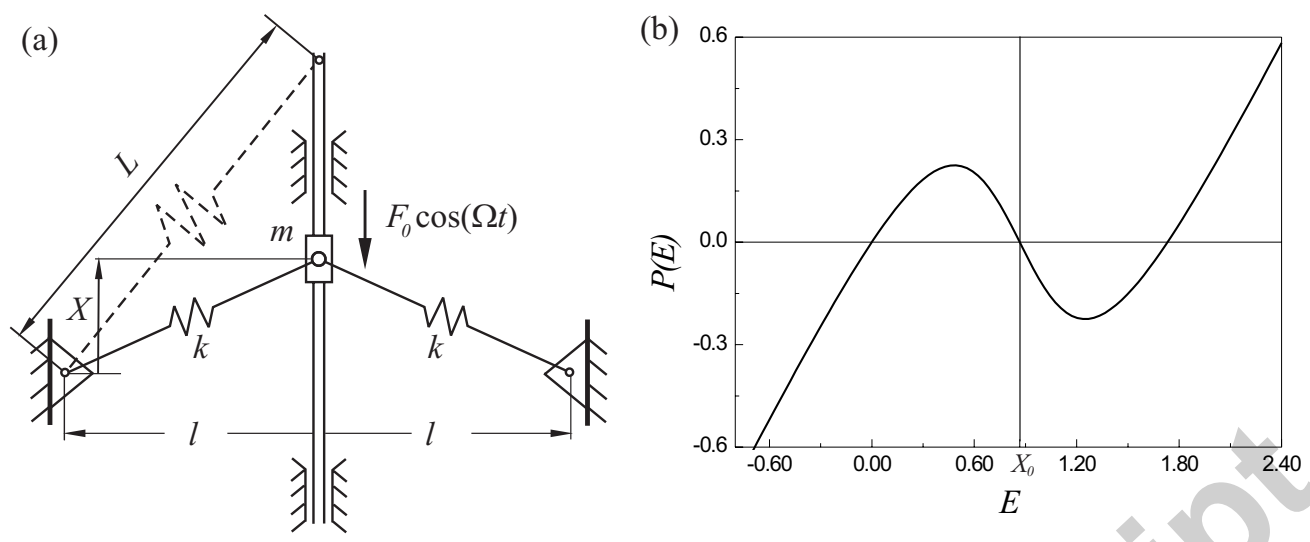

Figure 1: (a) A nonlinear oscillator with a lump mass and a pair of springs pinned to rigid supports, (b) function $P(E)$ for $L=1, k=\frac{1}{2}$ and $l=\frac{1}{2}$.

ical analysis carried out herein follows the mathematical tradition of nonlinear dynamics. They are, therefore, qualitatively typical and dynamically relevant. This conceptual model was used by Thompson \& Hunt, in [21], as an archetypal example of snap-through buckling. It is built using a mass, $m$ and two idealised elastic springs of stiffness $k$ by pinning them together at one end (the crown) and to two rigid foundations at the other as shown in Fig. 1a. Each spring has the initial (unstressed) length, $L$, and is assumed to remain straight under either tensile or compressive loading. The distance between these two rigid foundations (often called abutments, as in civil engineering) is written as $2 l$. Most of the time $L>l$ shall be taken, which gives one an unloaded symmetric arch of height

$$
X_{0}= \pm \sqrt{L^{2}-l^{2}}
$$

Here the minus sign corresponds to the inverted arch which is of course a valid (and stable) equilibrium state. Notice that if $L<l$, the unloaded springs will lie horizontally under tensile stress, and the behaviour will be that of a stretched elastic string.

The equilibrium response under a dead (prescribed and time independent) downwards vertical load, $P$, is written in terms of the downwards displacement of the crown, $E$ as

$$
P=-2 k\left(X_{0}-E\right)\left(1-\frac{L}{\sqrt{\left(X_{0}-E\right)^{2}+l^{2}}}\right),
$$

and shown in Figure 1b. Here the use of the downwards load against its corresponding displacement, $E$, gives us the conventional plot for an arch with stable regimes being easily identified as having positive slopes. Notice that $m$ is an inertial mass only, which does not contribute to the vertical loading on the arch. 


\section{Equations of motion for the limit case}

We are interested in the limit case shown in Figure 2a as $l$ decreases to zero, where the distance between the abutments, $2 l$, vanishes. In this case if the arch carries a compressive load, $P$, it will undergo a shortening, $s$, governed (we assume) by the linear constitutive law $P=2 k s$ and the stored strain-energy will be

$$
U=\frac{1}{2}(2 k) s^{2}
$$

This is a good approximation to reality when $s<<L$ where it corresponds to Hooke's Law for an elastic body. The approximation is reasonably physical under tensile loads when $P$ and $s$ are both negative. However, under compressive loads, this idealisation allows one to shrink the springs to zero length $(s=L)$ by applying the finite compressive load $P=2 k L$.

The load-deflection characteristic, $P(E)$ for the limit case of $l=0$ is shown in Figure $2 \mathrm{~b}$. We can now use this diagram to trace out the response of the limit arch with $l=0$. On the stable equilibrium path $\mathrm{ABC}$ the arch points upwards with the springs vertical, and its height varies linearly with the applied load. Meanwhile on the stable equilibrium path DEF the arch points downwards with the springs again vertical, and with its depth varying linearly with the applied load. Under a normal physical loading sequence under a slow increase of the dead load, $P$, the response would start at the unloaded state B and proceed quasistatically along the stable equilibrium path to $\mathrm{C}$. Here the system experiences a degenerate saddle-node fold, from which it would jump dynamically to an upsidedown configuration. The degenerate equilibrium path CD is unstable everywhere.

(a)

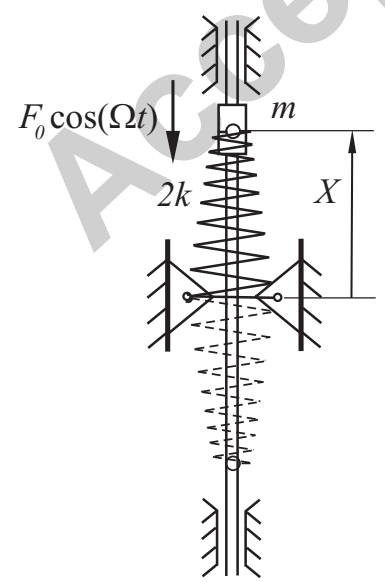

(b)

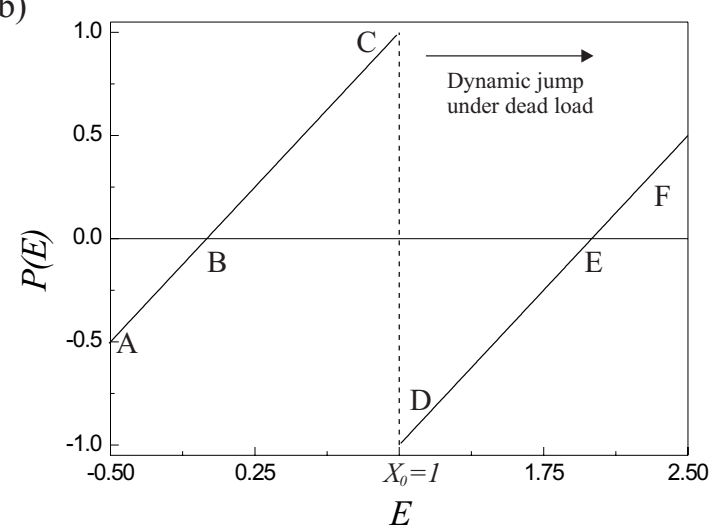

Figure 2: (a) The limit case, discontinuous oscillator with lump mass and a conical spring of stiffness $2 k$ capable of moving both above and below its pivot point, (b) limit case $(l=0)$ characteristics $P(E)$ for $k=\frac{1}{2}$ and $L=1$. 
Notice that this limiting system under consideration is illustrated in Figure 2(a) using a notional conical spring which can pass through itself. This helps us to visualise the snap-through process, though a conical spring would not be expected to accurately give the same discontinuous response as our arch (further research on this might be interesting). Assuming the mass to be hinged to the spring in such a way that it could move inside, we can imagine that under forcing it could pass through the fully compressed state at $X=0$, causing the spring to flip. Thus the resistance of the spring could force the mass to oscillate either around the upper or lower equilibrium positions (or both).

The equation of motion for this limit case can be written as:

$$
m \ddot{X}+2 k(X-\operatorname{sign}(X) L)=0, \operatorname{sign}(X)=\left\{\begin{array}{cl}
1, & \text { if } X>0 \\
0, & \text { if } X=0 \\
-1, & \text { if } X<0
\end{array}\right.
$$

System (4) can be made dimensionless by letting $\omega_{0}^{2}=\frac{2 k}{m}, x=\frac{X}{L}$ into

$$
\ddot{x}+\omega_{0}^{2}(x-\operatorname{sign}(x))=0 .
$$

For the system with damping and external harmonic excitation of amplitude $F_{0}$ and frequency $\Omega$, the equation of motion in the dimensionless form can be written as

$$
x^{\prime \prime}+2 \xi x^{\prime}+(x-\operatorname{sign}(x))=f_{0} \cos \omega \tau,
$$

where $\tau=\omega_{0} t, f_{0}=\frac{F_{0}}{m L \omega_{0}^{2}}, \omega=\frac{\Omega}{\omega_{0}}$ and $x^{\prime}=\frac{d x}{d \tau}$.

Figure 3a shows the discontinuous restoring force and the potential energy with double well for $\omega_{0}^{2}=1$. As can be seen the potential energy curve is non-smooth at $x=0$ due to the discontinuity.

\section{Hamiltonian dynamics}

In this section, standard dynamics governed by the hyperbolic structure associated with the stationary state of the double-well and the loss of local hyperbolicity at the saddle-like point is investigated for free oscillations. Then the weakly dissipative system without external excitation and, finally, the undampted forced oscillations are considered. The KAM (Kolmogorov-Arnold-Moser) structure [24] is shown with islands presenting quasi-periodic solutions and surrounded by a chaotic sea for the driven system without damping.

\subsection{Free oscillations}

The free undamped system (5) can be written as two first order differential equations by letting $x^{\prime}=y$ : 

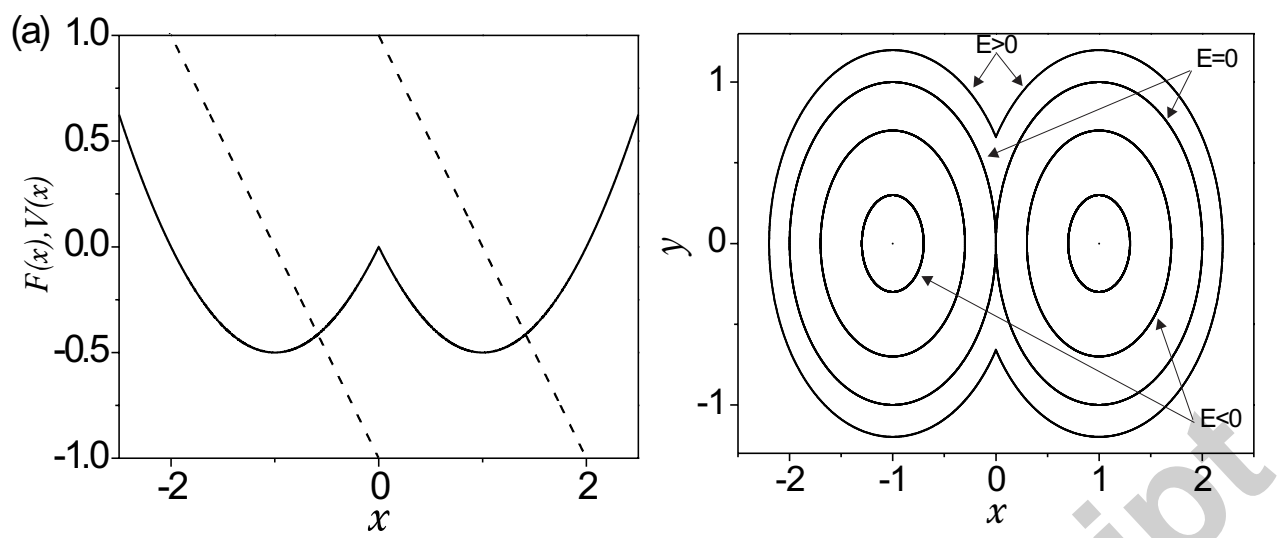

Figure 3: (a) Potential energy marked by solid curves and restoring force marked by dashed lines. (b) Phase portraits of the limit case oscillator for four different values of Hamiltonian function $H(x, y)=E$.

$$
\left\{\begin{array}{l}
x^{\prime}=y \\
y^{\prime}=-(x-\operatorname{sign}(x))
\end{array} .\right.
$$

The equilibria of the system are $(0,0)$ and $( \pm 1,0)$. Although the Jacobian matrix at point $(0,0)$ does not exist due to the discontinuity of the system at this point, the structure of the phase portrait near $(0,0)$ looks like a hyperbolic one, so it is referred to as the saddle-like singularity. However, the Jacobian matrix at $( \pm 1,0)$ does exist and it is calculated as

$$
J_{( \pm 1,0)}=\left(\begin{array}{cc}
0 & 1 \\
-1 & 0
\end{array}\right)
$$

with eigenvalues $\lambda_{1,2}= \pm i$. Thus the points $( \pm 1,0)$ are centre equilibria.

Multiplying both sides of the second equation of Eq. (7) by the first one, and integrating over $[0, x]$, one gets the Hamiltonian function as

$$
H(x, y)=\frac{1}{2} x^{2}+\frac{1}{2} y^{2}-|x| .
$$

The trajectories can be classified by taking different values of $H(x, y)=E$ and the corresponding phase portrait of the system is shown in Figure 3b. The dynamic behaviour is similar to that of the Duffing oscillator for the double well except for the homoclinic-like orbit. The Hamitonian (8) represents two centre points $( \pm 1,0)$ for $E=-\frac{1}{2}$. The trajectories for $-\frac{1}{2}<E<0$ are two families of circles with the centres at $(-1,0)$ and $(1,0)$ respectively, while the trajectories for $E>0$ are comprised of two large segments of circles with their centres located at $(-1,0)$ and $(1,0)$ connected at $x=0$. 
In the most interesting case, $E=0$, the phase portrait of the system is depicted by two circles centered at $( \pm 1,0)$ and connected to each other at the singularity point $(0,0)$, forming a special singular homoclinic-like orbit. The structure near this point looks like a saddle. As discussed above, the Jacobian at $(0,0)$ does not exist, and this isolated singularity has neither eigenvalue nor eigenvector. The two circles with exception of point $(0,0)$, are not the manifolds of the singularity, but the flow along these circles approaches the point $(0,0)$ as $x \rightarrow 0$ at the rate $d y / d x$ equal to infinity. The flow is trapped by the singularity when entering it in a finite time. This homoclinic-like orbit for $E=0$, shown in Figure 3b, can be described in a parametric form as

$$
\Gamma_{0}=\left\{\left(x_{ \pm}(\tau), y_{ \pm}(\tau)\right), \tau \in(-\pi, \pi)\right\} \bigcup\{(0,0)\}
$$

where $\left(x_{ \pm}(\tau), y_{ \pm}(\tau)\right)=( \pm 1 \pm \cos \tau, \mp \sin \tau)$.

\subsection{Dissipative oscillations}

In this subsection, the limit case oscillator with dissipation is investigated. For the case when $\xi \neq 0$ and $f_{0}=0$, Eq. (6) takes form

$$
x^{\prime \prime}+2 \xi x^{\prime}+(x-\operatorname{sign}(x))=0 .
$$

The damping ratio $\xi$ is always assumed to be small, $\xi<<1$. The equilibria of this damped system are the same as that of the system $(7)$. The equilibria $( \pm 1,0)$ of system (10) are stable spirals with the eigenvalues having negative real parts, $\lambda_{1,2}=-\xi \pm i \sqrt{1-\xi^{2}}$. The property of the saddle-like singularity $(0,0)$ remains unchanged as that of system (7).

The basins of attraction of the stable singularities $( \pm 1,0)$ are shown in Figure $4 \mathrm{a}$ and the basin boundary is plotted in Figure 4b. The point $(0,0)$ is located in the basin boundary. This boundary is the separatrix curve which separates the basins of attraction of equilibria $( \pm 1,0)$. Along this separatrix, the flow approaches the point $(0,0)$ as $x \rightarrow 0$ at the rate $d y / d x$ equal to infinity. The flow will be trapped by the singularity when entering it in finite time, which is similar with the undamped situation discussed in Section 4.1.

\subsection{Undamped forced oscillations}

For $\xi=0$ and $f_{0} \neq 0$, Eq. (6) takes the form

$$
x^{\prime \prime}+(x-\operatorname{sign}(x))=f_{0} \cos \omega \tau,
$$

which describes an undamped externally driven oscillator. This system is nonintegrable due to the explicit presence of time $\tau$ in the forcing term. The timedependent Hamiltonian function is obtained and written as

$$
H_{\tau}(x, y, \tau)=\frac{1}{2} y^{2}+\frac{1}{2} x^{2}-|x|-f_{0} x \cos \omega \tau .
$$



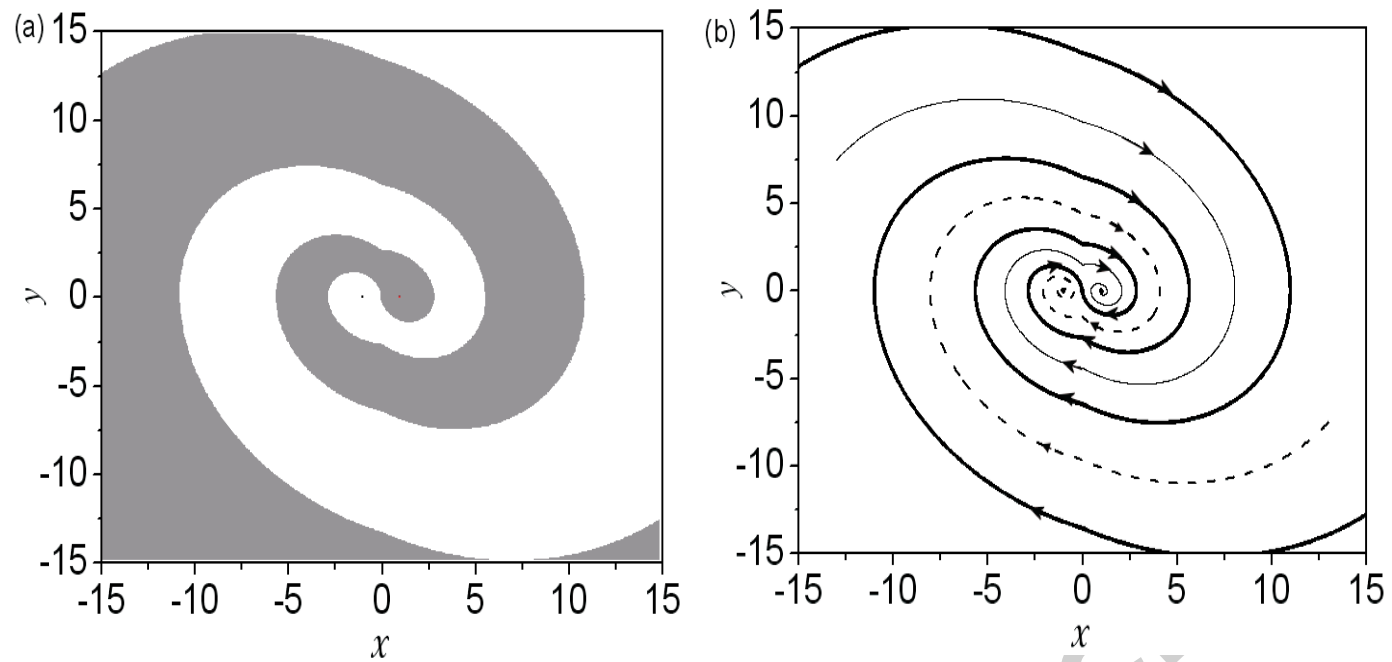

Figure 4: Damped behaviour for $\xi=0.2$. (a) The basins of attraction are shown in grey for the spiral $(1,0)$ and in white for $(-1,0)$; (b) the separatrix on the border of the basins of attraction, is marked as the bold solid curve, and the trajectories converging to the stable spirals $(1,0)$ and $(-1,0)$ are shown by thin solid and dashed curves respectively.

Numerical investigations of the system described by Eq.(11) have been carried out using semi-analytical method proposed in [20]. The results of this study are presented next in the form of Poncaré sections based on the forcing frequency and taken at zero phase angle and trajectories on the phase plane.

Figure 5 demonstrates Poincaré section calculated for $\xi=0, f_{0}=0.8$ and $\omega=$ 1.05. In this Poincare section, the KAM structure, $[24,25]$, is presented with a series of periodic fixed points and their surrounding islands, and also the chaotic orbit connecting these islands. Among these chaotic orbits, there exists a special one identified as a chaotic sea [25, 26, 27], which fills the finite area and connects the isolated islands encircling associated series of fixed points. As can be seen from this figure, KAM curves and another series of fixed points (a pair of period6, a pair of period- 7 and period-13 marked by arrows) and the associated quasiperiodic islands with the chaotic orbits connecting these islands can be found outside the finite region filled by the special chaotic orbit.

Figure 6a shows the dynamics within the finite area filled by the special chaotic orbit. This orbit connects quasi-periodic islands, [27], encircling a number of periodic fixed points. A pair of period-2, a pair of period-5, one period-9 and one period-11 orbits were found co-existing with the chaotic orbit in this region. Thus as can be seen the dynamical structure of this region is formed by the chaotic sea together with periodic and associated quasi-periodic solutions.

Figure $6 \mathrm{~b}$ and $6 \mathrm{c}$ present details of the Poincaré section for two rectangular areas marked in Figure 5. Again a number of periodic fixed points with associated 


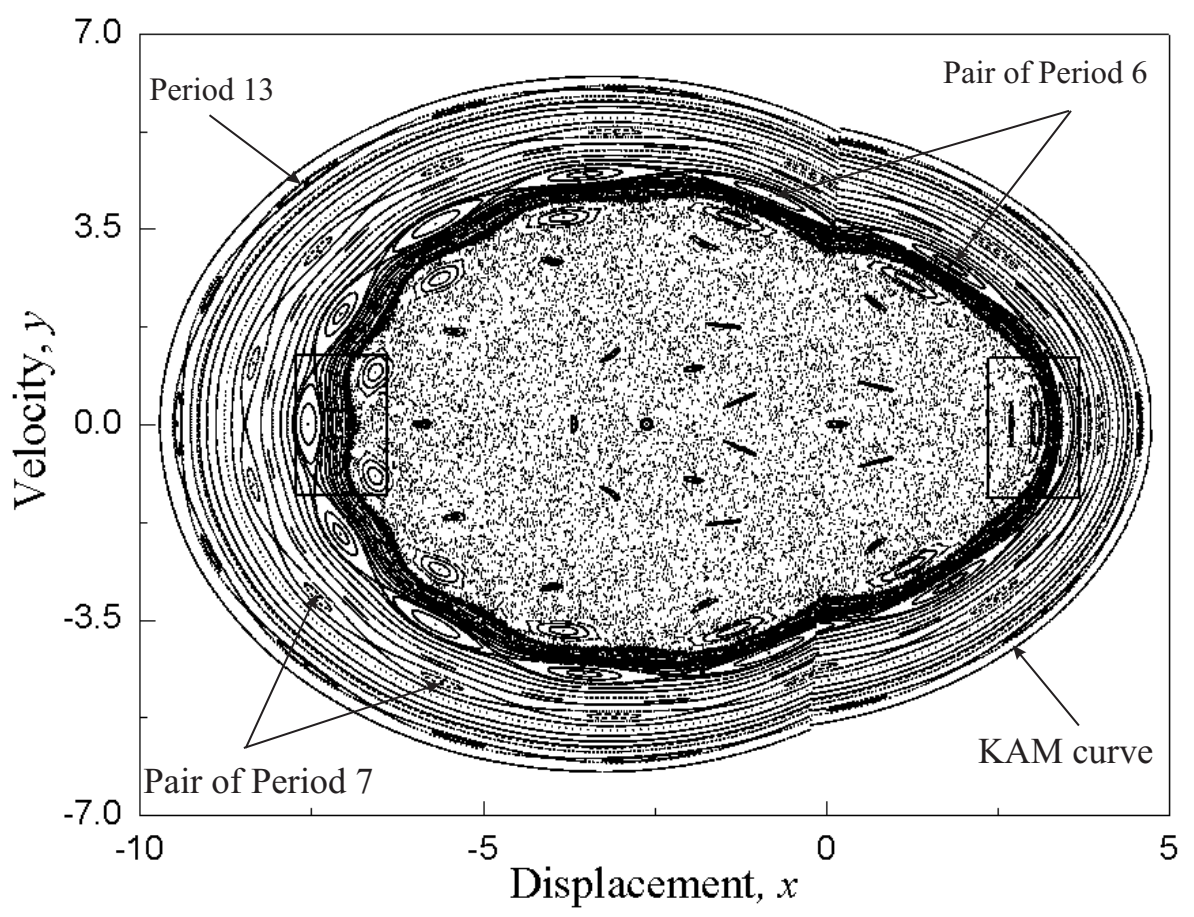

Figure 5: Global KAM structure on Poincaré section with KAM orbits, series of fixed points with the associated quasi-periodic islands and also the chaotic sea chaining these islands. KAM curve and series of fixed points of period 13, pair of period 6 and pair of period 7 fixed points are marked.

quasi-periodic islands surrounded by chaotic orbits can be seen in these figures. Period-5, period-6, period-11, period-17 and period-23 orbits are marked in Figure $6 \mathrm{~b}$ whereas period- 6 , period- 11 , period- 17 and period- 23 orbits are specified in Figure 6c. Figure 6d shows the details of the chaotic orbit connecting the island chain in the rectangle area marked on Figure 6c.

Other examples of chaotic seas and co-existing periodic orbits found under varying the external frequency $\omega$ are presented in Figure 7 for $f_{0}=0.8$. In Figure 7 a obtained at $\omega=1.3$, four different period- 5 orbits are shown in gray, light gray, green, and blue colours respectively. Figure $7 \mathrm{~b}$ obtained at $\omega=\frac{1}{3}$ presents a pair of period-1 orbits shown in gray and light gray colours, a pair of period-4 orbits shown in dark gray and yellow colours and period-5 orbit shown in red colour. Trajectories and Poincaré sections for one of the period-5 orbits shown in Figure $7 \mathrm{a}$ and one of the period- 1 orbits shown in Figure $7 \mathrm{~b}$ are displayed on the phase plane in Figures $7 \mathrm{c}$ and $7 \mathrm{~d}$ respectively. 
(a)

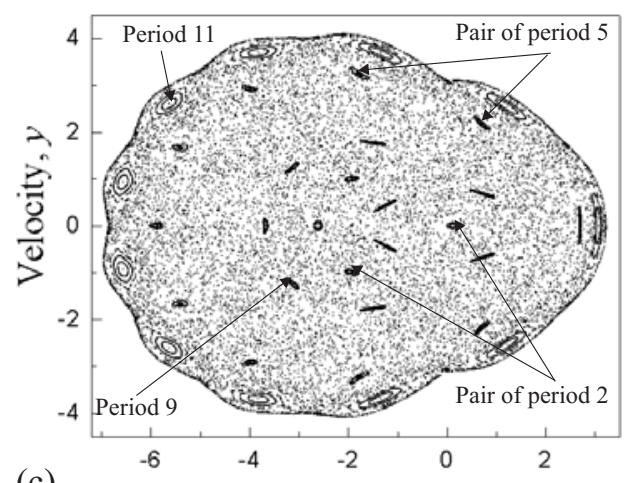

(c)

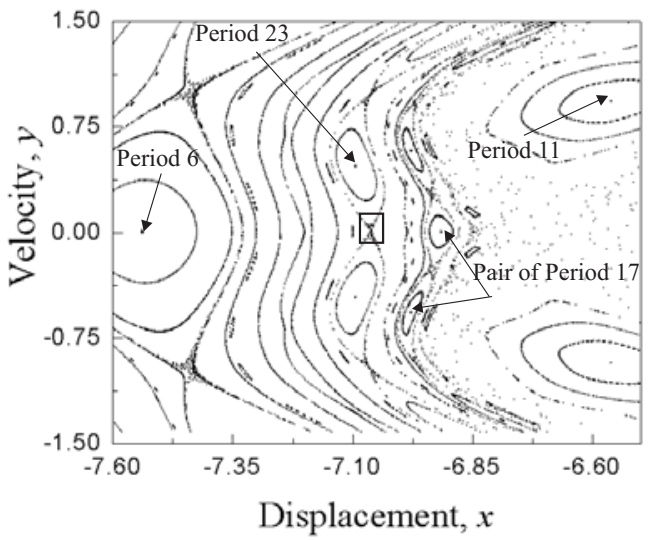

(b)

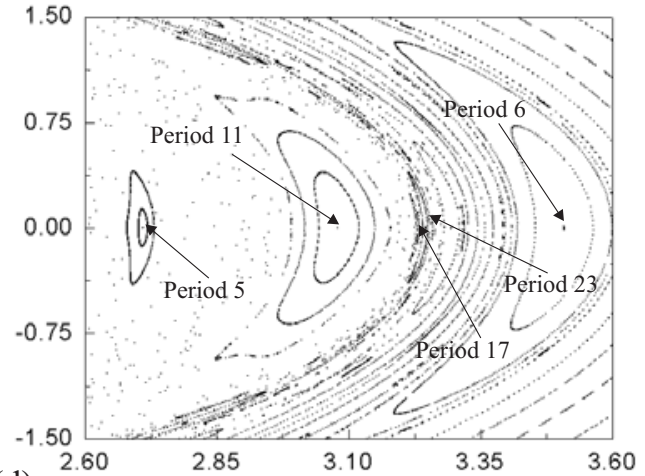

(d)

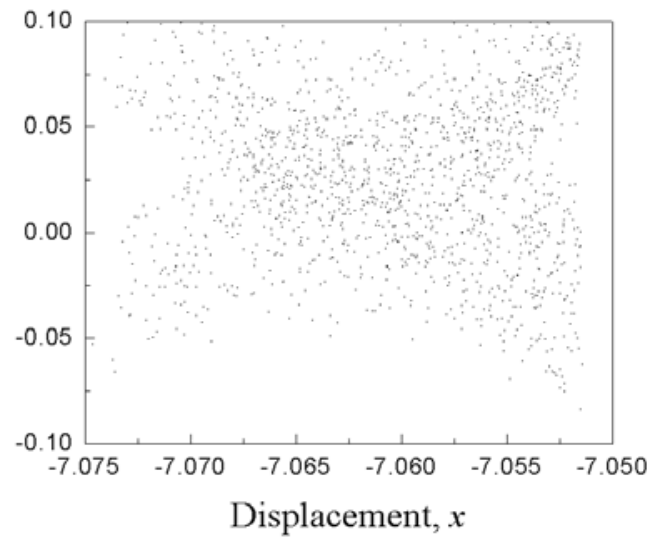

Figure 6: Details of Figure 5. (a) The chaotic trajectory filling the finite region and connecting islands encircling a series of fixed points; (b) details of the structure in the right rectangle area marked on Figure 5; (c) details of the structure in the left rectangle area marked on Figure 5; (d) chaotic orbit connecting the quasi-periodic islands chain in the rectangle area marked on Figure 6c.

\section{Driven system with weak dissipation}

In this Section, numerical analysis is carried out to investigate the dynamics for the system with weak dissipation and external excitation. The semi-analytical method [20] is used in the following analysis to overcome the numerical difficulties caused by the discontinuity. All the bifurcation diagrams are plotted with 5000 cycles of pre-iterates and 200 samples for each calculation.

\subsection{Influence of weak dissipation}

As discussed in previous sections, the discontinuous system without dissipation exhibits periodic solutions and the associated quasi-periodic solutions. In this subsection, we investigate the behaviour of these solutions in the presence of weak dissipation. 
(a)

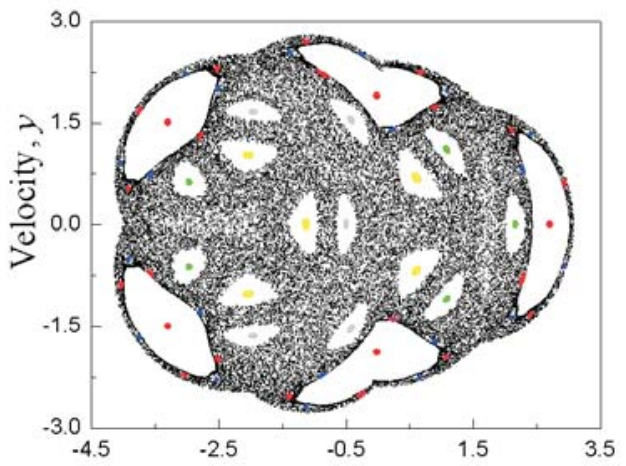

(c)

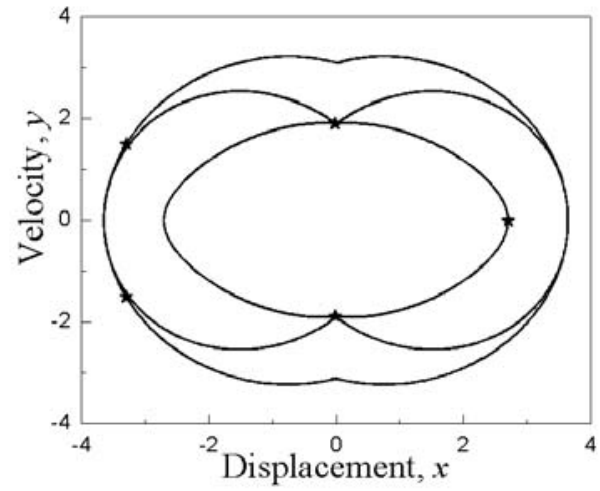

(b)

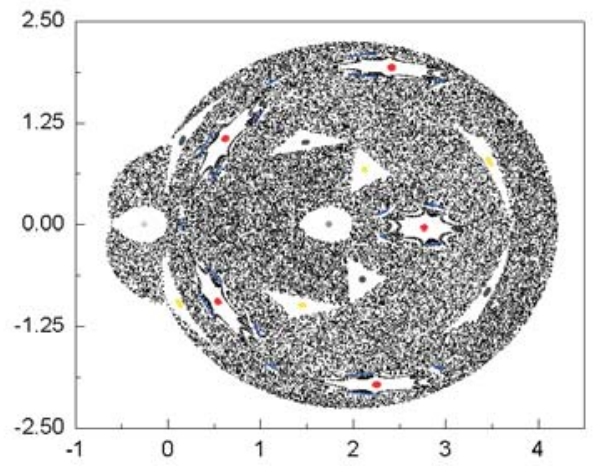

(d)

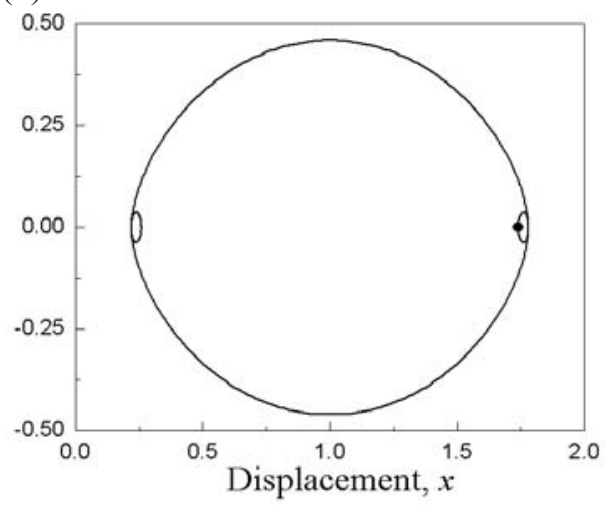

Figure 7: Chaotic seas and periodic solutions for $f_{0}=0.8$. (a) For $\omega=1.3$, four different period-5 orbits are shown in gray, light gray, green and yellow colours respectively; (b) for $\omega=\frac{1}{3}$, a pair of period-1 orbits are marked by gray and light gray points, a pair of period-4 orbits by dark gray and yellow points, and period- 5 orbit is shown in red; (c) and (d) are the trajectories and the Poincaré sections for one of the period- 5 orbits shown in Figure $7(\mathrm{a})$ and one of the period-1 orbits shown in Figure 7(b), respectively.

Bifurcation diagrams are given for velocity $y$ versus the weak dissipation $\xi$ in Figs. $8 \mathrm{a}$ and $8 \mathrm{~b}$ starting from quasi periodic solutions for $\xi=0$. It is known that introduction of an arbitrary small dissipation destroys the quasi-periodic solutions, and for the considered system as soon as $\xi \neq 0$ they become periodic solutions of the same period. Therefore these diagrams show the bifurcation from corresponding periodic solutions to chaotic solutions as the dissipation increases.

As can be seen from Figure 6a a pair of period-2, a pair of period-5, one period9 and one period-11 orbits and associated with them quasi-periodic orbits were found co-existing with the chaotic sea in this region. The bifurcations of three of these quasi-periodic solutions generated corresponding periodic solutions in the presence of damping as shown in Figure 8a. In this figure three diagrams are plotted showing bifurcations of originally period-2 orbits (in blue), period- 
(a)

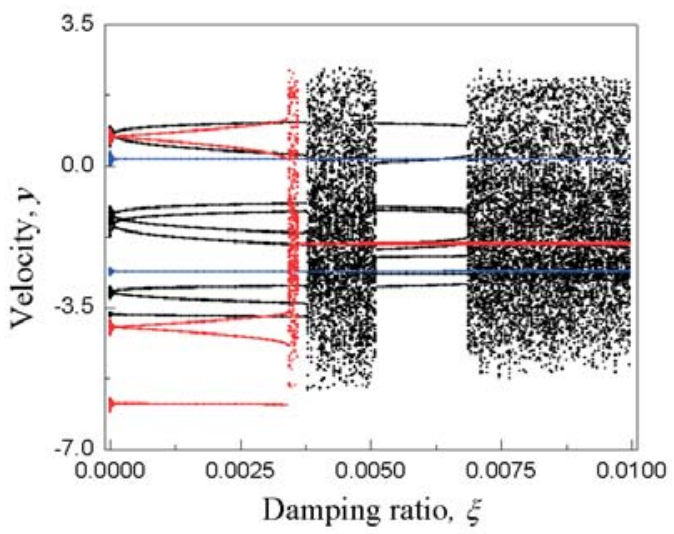

(b)

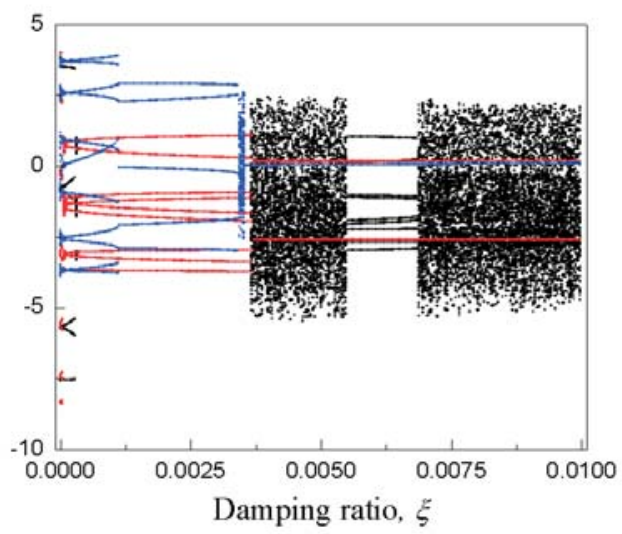

Figure 8: Bifurcation diagrams showing the stroboscopically-sampled velocity $y$ versus damping ratio $\xi$ for $f_{0}=0.8, \omega=1.05$. (a) The black points indicate an originally period-9 orbit and its behaviour as $\xi$ increases, the blue points show bifurcations of an originally period-2 orbit and the red points present bifurcations of an originally period-5 orbit; (b) The black points show the bifurcations of an originally quasi-periodic orbit of period-6, the blue points an originally quasiperiodic orbit of period-11 and the red points an originally quasi-periodic orbit of period-13.

5 orbit (in red) and period-9 orbit (in black). Figure 9 shows the co-existing periodic orbits for $\xi=0.001, f_{0}=0.8, \omega=1.05$, i.e. a pair of period-2 solutions (given in Figure 9a), a pair of period-5 solutions (Figure 9b), period-9 solution (Figure 9c) and period-11 solution (Figure 9d). Figure 9e presents the Poincaré section for co-existing periodic orbits. This analysis allows one to conclude that the co-existence of periodic solutions within the finite region as shown in Figure $6 \mathrm{a}$ remains unchanged as one goes from the Hamiltonian to the dissipative dynamics by the addition of weak dissipation [28, 29].

A number of quasi-periodic solutions co-exist also outside the finite area filled by the chaotic sea as shown in Figures $6 \mathrm{~b}$ and $6 \mathrm{c}$. Their behaviour in the presence of weak dissipation was also examined and some of the results are given in Figure 8b, which presents bifurcation of originally quasi-periodic orbits of period-6 (in black), of period-11 orbit (in blue) and of period-13 orbit (in red). It was found that the periodic solutions existing for $\xi=0$ outside of the finite area filled by the chaotic sea together with the associated quasi-periodic solutions are nonpersistent in the presence of damping.

In addition to the periodic bifurcations, other periodic windows and chaotic regions can be seen from Figures $8 \mathrm{a}$ and $8 \mathrm{~b}$. There are two main intervals in which chaotic motions occur. The chaotic attractors for $f_{0}=0.8, \omega=1.05$, are presented in Figure 10a for $\xi=0.005$ and 10b for $\xi=0.01$, respectively.

Our numerical studies also suggest the presence of long chaotic transients before 
(a)
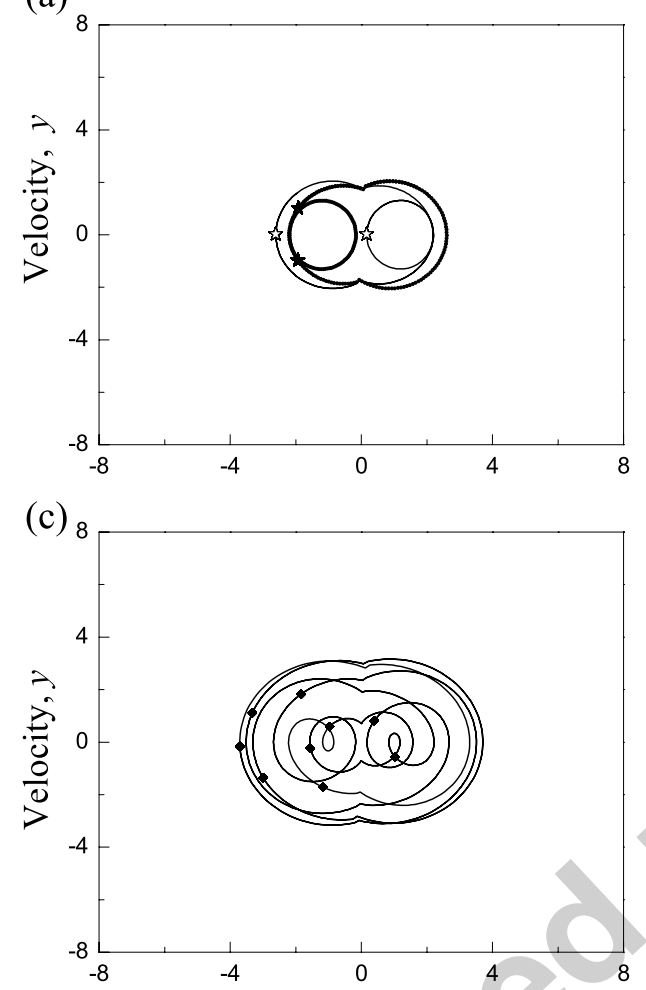

(e)

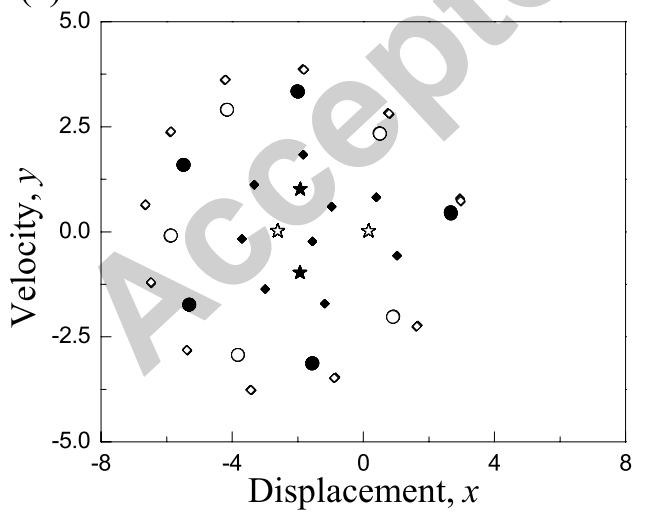

(b)

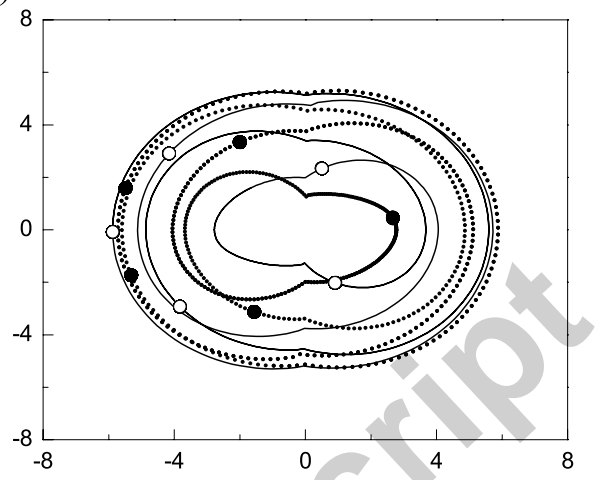

(d)

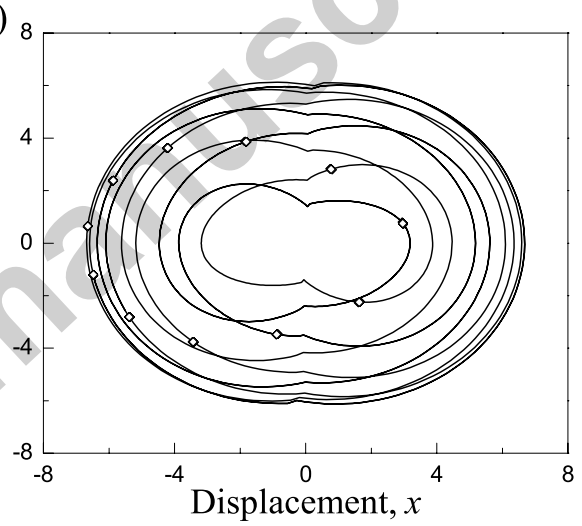

Figure 9: The co-existence of periodic orbits for $\xi=0.001, f_{0}=0.8, \omega=1.05$. (a) A pair of period-2 solutions; (b) a pair of period-5 solutions; (c) period9 solution; (d) period-11 solution; (e) Poincaré section for all the co-existing periodic solutions. 
the trajectory settles down to a periodic solution. This behaviour can be characterized by chaotic saddles, see [30, 31, 32]. The chaotic transient and the final period 2 attractor are shown in Figure 11a, for $\xi=0.0035$. Figure 11b shows the velocity $y$ as function of time $\tau$ presenting the transient process from the chaotic saddle to the period-2 orbit. Similar behaviour was observed by Thompson and Ghaffari, see also Thompson and Stewart, [33, 34]. The latter arose in a linear oscillator with impacts, [34], which has apparent similarities with the present model.

(a)

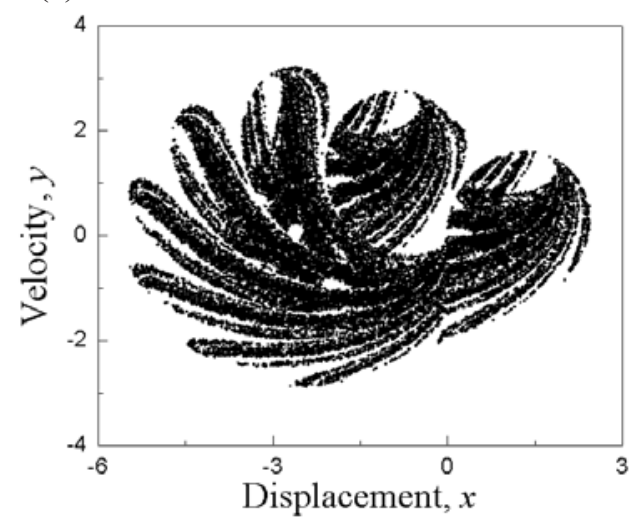

(b)

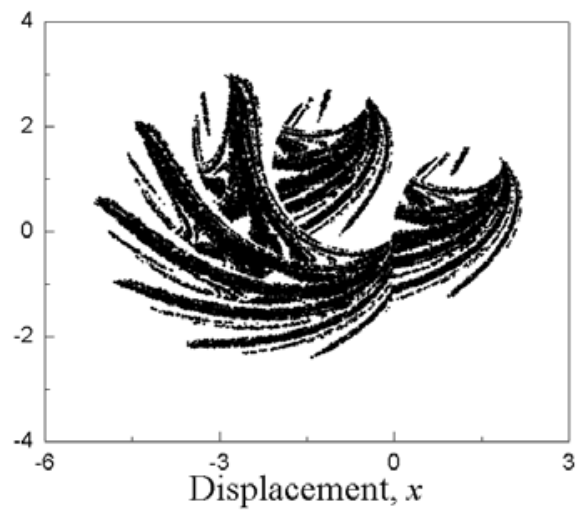

Figure 10: Chaotic orbits in the presence of weak dissipation obtained for $f_{0}=$ $0.8, \omega=1.05$ at (a) $\xi=0.005$; (b) $\xi=0.01$.

(a)

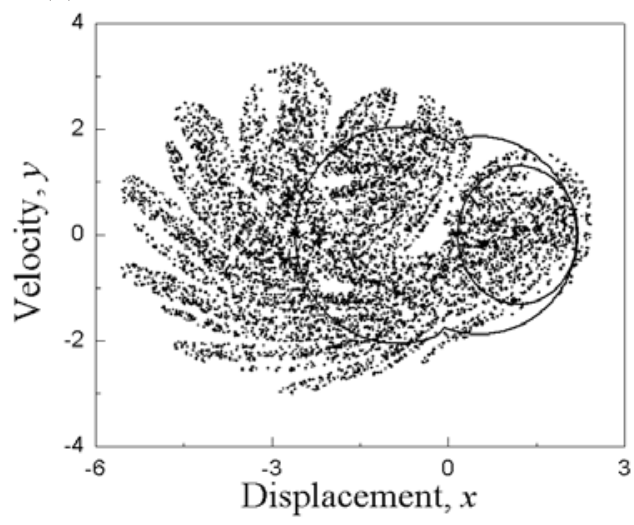

(b)

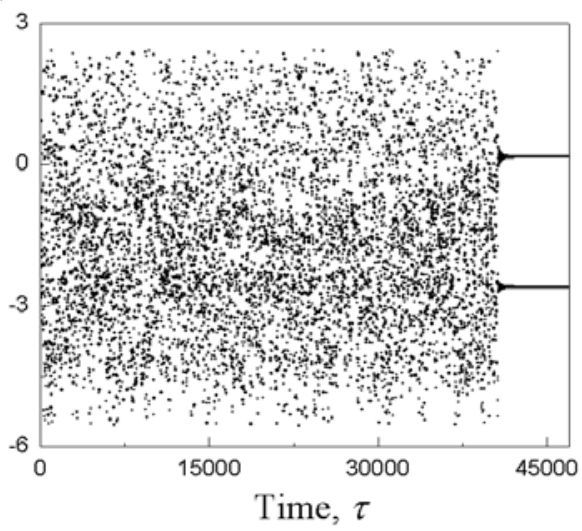

Figure 11: State space plots for $f_{0}=0.8, \omega=1.05$ and $\xi=0.0035$. (a) Poincaré section of the chaotic saddle and trajectory of the resulting period-2 orbit; (b) stroboscopic velocity $y$ versus time $\tau$ exhibiting the long transient before settling down to a period-2 orbit. 
(a)

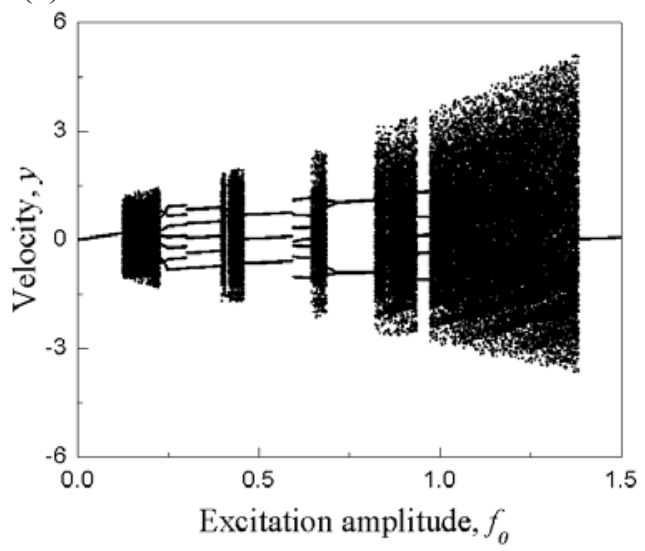

(b)

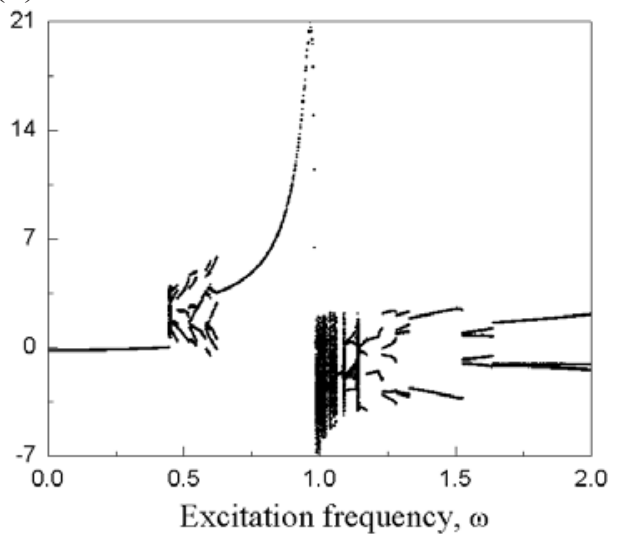

Figure 12: Bifurcation diagrams: (a) for $y$ versus $f_{0}$ for $\omega=1.05$ and $\xi=0.01$, (b) for $y$ versus $\omega$ for $f_{0}=0.8$ and $\xi=0.001$.

\subsection{Influence of external forcing}

Here the influence of forcing on the dynamic behaviour of the system in the presence of weak dissipation is examined. The bifurcation diagrams and the attractors are displayed to show the results.

Figure 12a shows the bifurcation diagram of the system under varying amplitude of the external forcing $f_{0}$ for $\omega=1.05$ and $\xi=0.01$. From this bifurcation diagram, multiple periodic windows and chaotic regions can be seen. As an example, the periodic doubling leading to chaos is presented in Figure 13 for $\omega=1.05$ and $\xi=0.01$. Figure 13a to Figure 13c shows the periodic trajectories on the phase plane and the corresponding Poincaré sections, i.e. period-2 orbit at $f_{0}=0.75$ (Figure 13a), period- 4 orbit at $f_{0}=0.7$ (Figure 13b), and period- 8 orbit at $f_{0}=0.6875$ (Figure 13c). Finally Figure 13d shows the chaotic attractor at $f_{0}=0.675$.

The influence of the external frequency $\omega$ is shown in Figure 12b where the bifurcation diagram for the velocity $y$ versus $\omega$ is presented for $f_{0}=0.8$ and $\xi=0.001$. From this bifurcation diagram, multiple periodic windows and chaos can be seen. Some special periodic orbits are shown in the following figures.

Figures 14a and 14b shows the trajectories of period-3 and period-2 orbits, for $\omega=$ 0.5 and $\omega=0.56$, respectively. Figures $15 \mathrm{a}$ and $15 \mathrm{~b}$ present trajectories of period7 and period- 3 orbits, for $\omega=1.125$ and $\omega=1.64$, respectively and Figures 16a and $16 \mathrm{~b}$ display the trajectories of period- 2 and period-3 orbits for $\omega=1.4$ and $\omega=2$, respectively. The black stars in the figures mark the corresponding Poincaré points. 


\section{Conclusions}

In this paper, we have investigated the behaviour of the limit discontinuous case of the archetypical SD oscillator introduced to study transition from smooth to discontinuous dynamics. It has been shown that the system exhibits not only the standard dynamics associated with the equilibrium state of the double-well but also the dynamics associated with the loss of the local hyperbolicity due to the discontinuity resulting in a homoclinic-like structure. The velocity flow along this homoclinic-like orbit approaches the saddle-like equilibrium $(0,0)$ at the rate $d y / d x= \pm \infty$ as $d x \rightarrow 0$.

The KAM structure in the Poincaré section has been constructed for the driven system without dissipation with the generic KAM curves and the series of periodic orbits with the surrounded island chains connected by chaotic orbits. Chaotic seas have been depicted with a special chaotic trajectory filling a finite area together with several series of periodic orbits and the surrounded islands.

The results obtained in this paper showed that the co-existence of these periodic solutions remains unchanged in the presence of weak dissipation. The relevant dynamics has also been depicted for this discontinuous system with weak dissipation under external excitation exhibiting period doubling leading to chaos and coexistence of attractors.

\section{Acknowledgement}

The first two authors acknowledge the financial support from EPSRC and the Scottish Enterprise.

\section{References}

[1] Filippov, A.F. Differential equations with discontinuous right-hand sides, Mathematics and Its Applications, Kluwer Academic, Dordrecht, 1988.

[2] Feigin, M.I. Forced vibrations of nonlinear systems with discontinuities, Nauka, Moscow, 1994 (in Russian).

[3] Leine, R.I. and Van Campen, D.H. Discontinuous bifurcations of periodic solutions, Mathematical and Computer Modelling, 36, 2002: 259-273.

[4] Leine, R.I., Van Campen, D.H. and Van de Vrande, B.L. Bifurcations in Nonlinear Discontinuous Systems, Nonlinear Dynamics, 23, 2000: 105-164.

[5] Leine, R.I., Van Campen, D.H., De Kraker, A. and Van den Steen, L. Stick-slip vibrations induced by alternate friction models, Nonlinear Dynamics, 16(1), 1998: 41-54.

[6] Filippov, A. F., Differential equations with discontinuous right-hand side, American Mathematical Society Translations, Series 2, 42, 1964:199-231. 
[7] Foong, C.H., Pavlovskaia, E., Wiercigroch M. and Deans, W.F., Chaos caused by fatigue crack growth, Chaos, Solitons and Fractals 16, 2003: 651-59.

[8] Ouyang, H., Mottershead, J.E., Cartmell, M.P. and Brookfiled, D.J. Frictioninduced vibration of an elastic slider on a vibrating disk, International Journal of Mechanical Sciences, 41(3), 1999: 325-336

[9] Wiercigroch, M. Modelling of dynamical systems with motion dependent discontinuities, Chaos Solitons \& Fractals, 11(15), 2000: 2429-2442.

[10] Pavlovskaia, E., Wiercigroch, M. Periodic solution finder for an impact oscillator with a drift, Journal of Sound and Vibration, 267(4), 2003: 893-911.

[11] Xu L., Lu M.W., Cao Q. Nonlinear vibrations of dynamical systems with a general form of piecewise-linear viscous damping by incremental harmonic balance method, Physics Letters A, 301(1-2), 2002: 65-73

[12] Cao, Q., Xu, L., Djidjeli, K., Price, W.G. and Twizell, E.H. Analysis of perioddoubling and chaos of a non-symmetric oscillator with piecewise-linearity, Chaos Solitons \& Fractals, 12(10), 2001: 1917-1927.

[13] Di Bernardo, M., Kowalczyk, P., Nordmark, A. Sliding bifurcations: a novel mechanism for the sudden onset of chaos in dry friction oscillators, International Journal of Bifurcation and Chaos, 13(10), 2003: 2935-2948.

[14] Di Bernardo, M., Garofalo, F., Iannelli, L, Vasca, F., Bifurcations in piecewisesmooth feedback systems, International Journal of Control 75(16-17), 2002: 12431259 .

[15] Di Bernardo, M., Kowalczyk, P., Nordmark, A. Bifurcations of dynamical systems with sliding: derivation of normal-form mappings, Physica D, 170(3-4), 2002: 175205.

[16] Banerjee, S. and Grebogi, C. Border collision bifurcations in two-dimensional piecewise smooth maps, Physical Review E, 59(4), 1999: 4052-4061

[17] Banerjee, S., Karthik, M. S., Yuan G. H. and Yorke, J. A. Bifurcations in onedimensional piecewise smooth maps - theory and applications in switching circuits, IEEE Transactions on Circuits \& Systems -I, 47(3), 2000: 389-394.

[18] Whiston, G.S. Singularities in vibro-impact dynamics, Journal of Sound and Vibration 152(33), 1992: 427-460.

[19] Cao, Q., Wiercigroch, M., Pavlovskaia, E.E., Grebogi, C. and Thompson, J.M.T. An archetypal oscillator for smooth and discontinuous dynamics, Physical Review E 74, 2006: 046218.

[20] Cao, Q., Wiercigroch, M., Pavlovskaia, E.E., Grebogi, C. and Thompson, J.M.T. Piecewise linear approach to an archetypal oscillator for smooth and discontinuous dynamics, Phil. Trans. R. Soc. A (accepted). 
[21] Thompson, J.M.T., Hunt, G.W. A general theory of elastic stability, John Wiley \& Sons, London, 1973.

[22] Pippard, A.B. The elastic arch and its modes of instability, European Journal of Physics, 11(6), 1990: 359-365.

[23] Savi, M.A. and Pacheco, P.M.C.L. Transient chaos in an elasto-plastic beam with hardening. Journal of Brazilian Society of Mechanical Sciences and Engineering, 25(2), 2003: 189-193.

[24] Lichtenberg, A.J. and Lieberman, M.A. Regular and chaotic dynamics, SpringerVerlag, New York, 1992.

[25] Chernikov, A. A., Sagdeev, R.Z., Usikov, D.A., Zakharov, M.Yu, and Zaslavsky, G.M., Minimal chaos and stochastic webs, Nature, 326, 1987: 559-563.

[26] Pekarsky, S. and Rom-Kedar, V. Uniform stochastic web in low-dimensional hamiltonian systems. Phys. Lett. A225, 1997: 274-286.

[27] Luo, A.C.J. Chaos and quasi-periodic motions on the homoclinic surface of nonlinear hamiltonian systems with two degrees of freedom, Journal of Computational and Nonlinear Dynamics, 1(2), 2006: 135-142.

[28] Feudel, U., Grebogi, C., Hunt, B.R. and Yorke, J. A. Map with more than 100 coexisting low-period periodic attractors, Physical Review E 54, 1996: 71 - 81.

[29] Feudel, U. and Grebogi, C. Multistability and the control of complexity, Chaos: an Interdisciplinary Journal of Nonlinear Science 7(4), 1997: 597-604.

[30] Lai, Y.C. and Winslow R.L. Geometric properties of the chaotic saddle responsible for supertransients in spatiotemporal chaotic systems, Physical Review Letters, 74(26), 1995: 5208-5211.

[31] Tel, T. Characteristic exponents of chaotic repellers as eigenvalues, Physics. Letters. A, 119(2), 1986: 65-68.

[32] Grebogi C., Ott E. and Yorke J.A. Crises, sudden changes in chaotic attractors, and transient chaos, Physica D, 7(1-3), 1983: 181-200.

[33] Thompson, J.M.T. and Ghaffari, R., Chaotic dynamics of an impact oscillator, Physical Review A, 27(3), 1983: 1741-1743.

[34] Thompson, J.M.T. and Stewart, H. B. Nonlinear Dynamics and Chaos (2nd edition), Wiley \& Sons, London, 2002. 


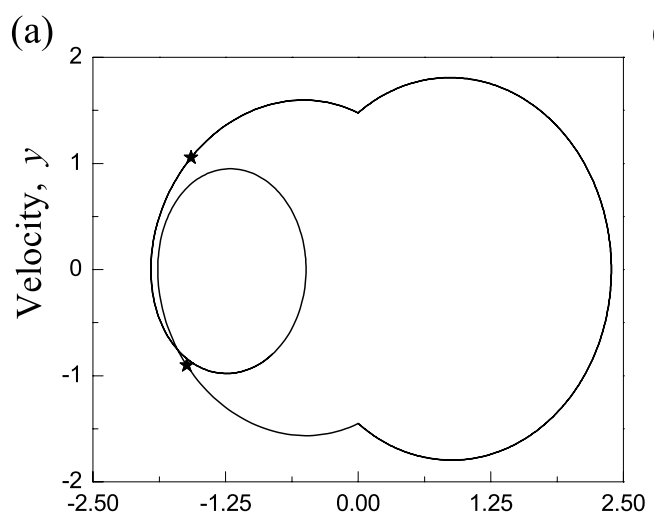

(b)

(c)
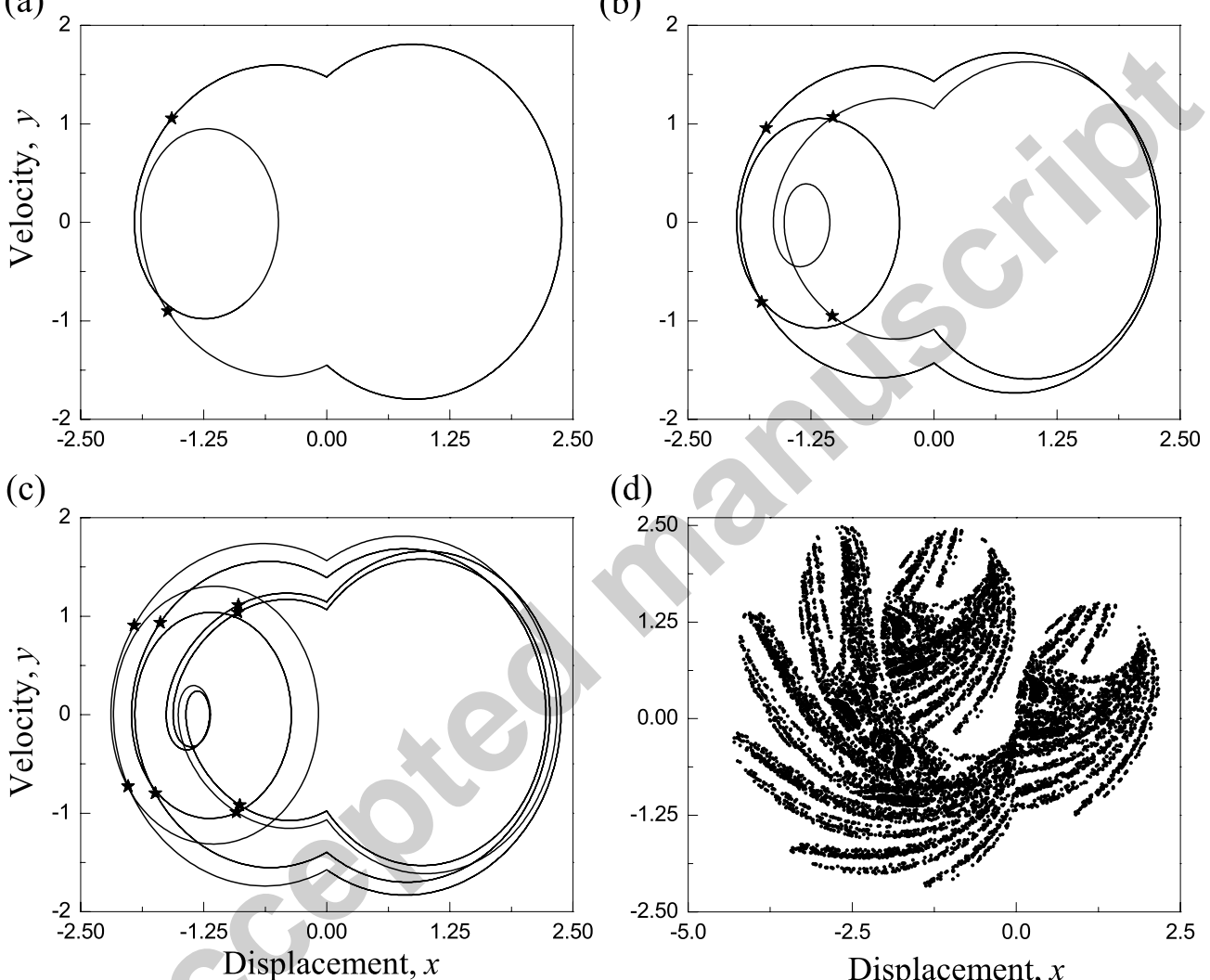

(d)

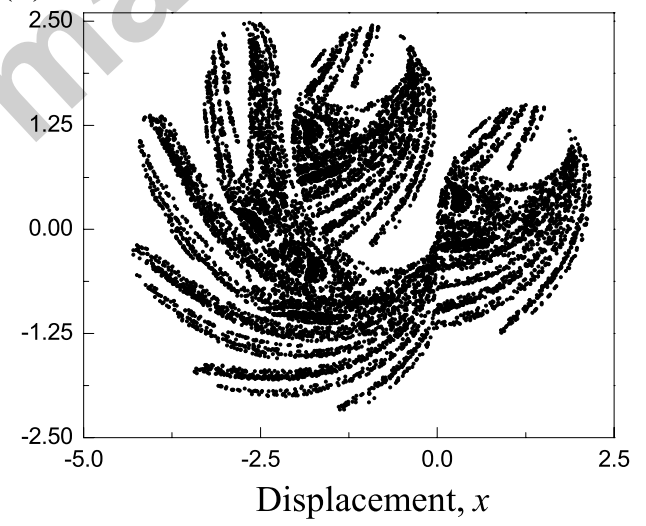

Figure 13: Period doubling bifurcation leading to chaos for $\omega=1.06066$ and $\xi=0.01$ : trajectories on the phase plane with Poincaré points for (a) period2 orbit for $f_{0}=0.75$, (b) period-4 orbit for $f_{0}=0.7$, (c) period-8 orbit for $f_{0}=0.6875$, and (d) Poincaré map of the chaotic attractor arising from period doubling for $f_{0}=0.675$. 

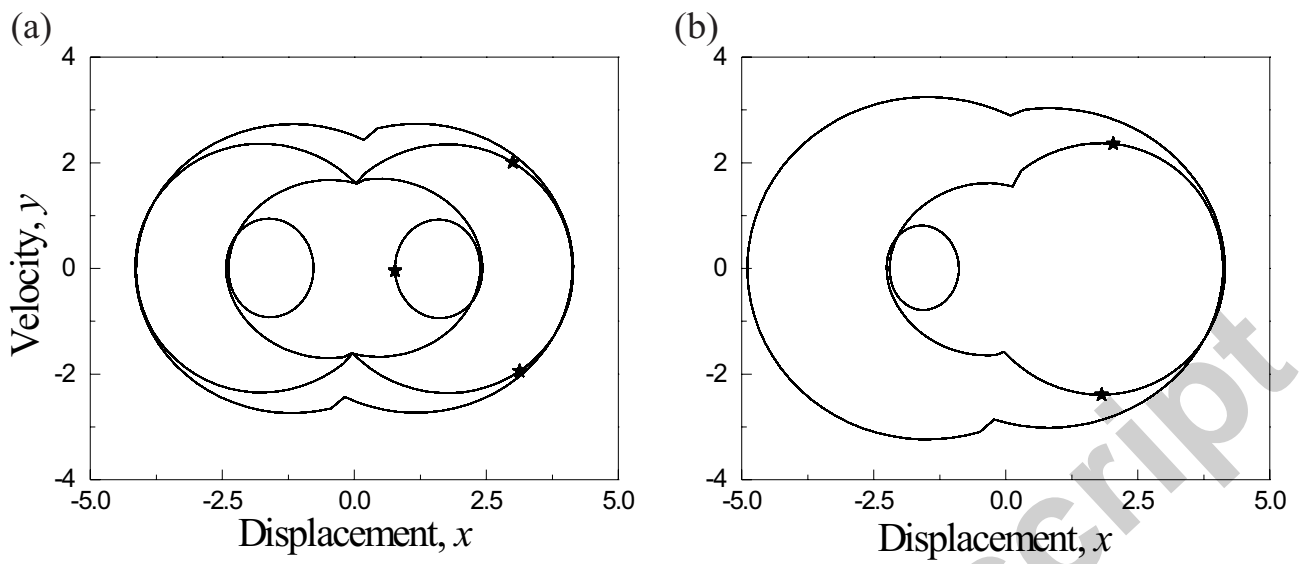

Figure 14: Periodic solutions for $f_{0}=0.8$ and $\xi=0.001$ (a) period-3 orbit for $\omega=0.5$ and (b) period-2 orbit for $\omega=0.56$.
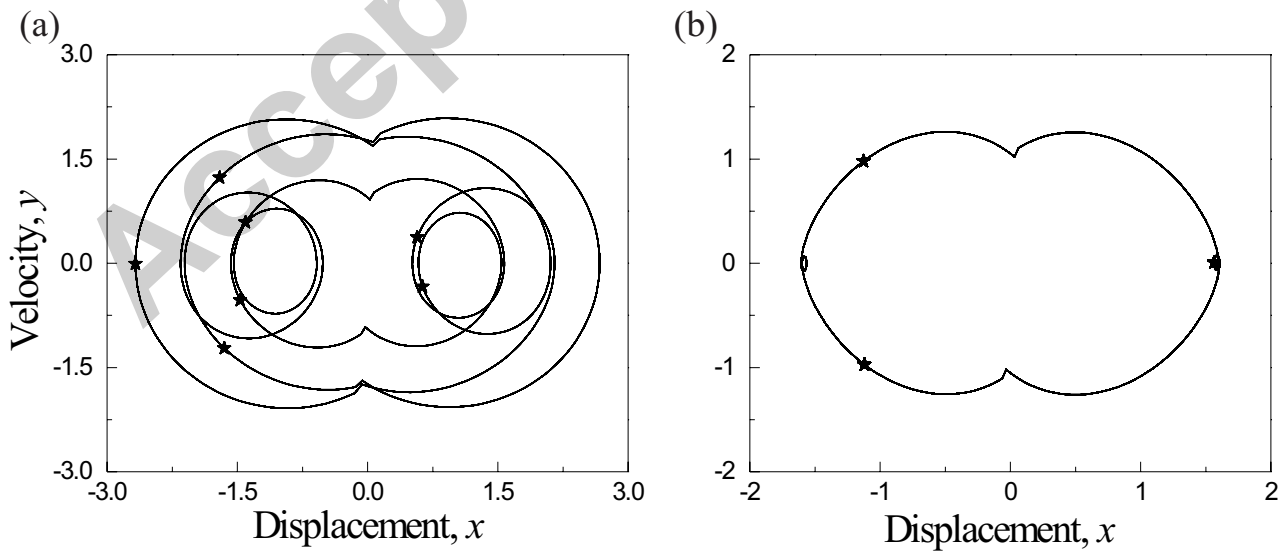

Figure 15: Periodic solutions for $f_{0}=0.8$ and $\xi=0.001$ : (a) period-7 orbit for $\omega=1.125$ and (b) period-3 orbit for $\omega=1.64$. 
(a)

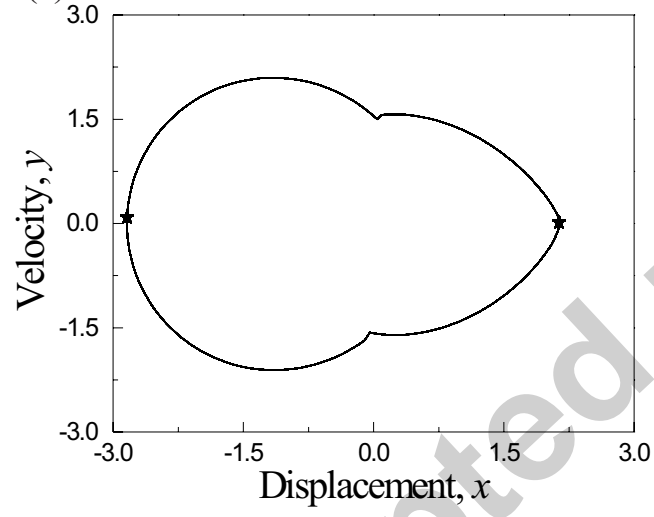

(b)

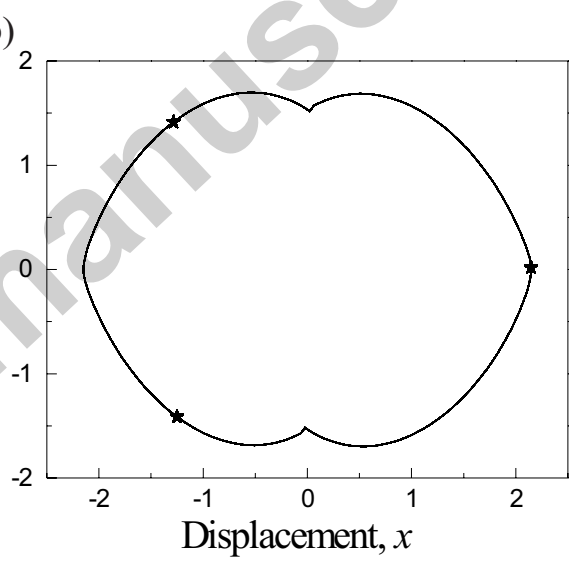

Figure 16: Periodic solutions for $f_{0}=0.8$ and $\xi=0.001$ : (a) period-2 orbit for $\omega=1.4$ and (b) period-3 orbit for $\omega=2.0$. 